\title{
Rapid access neurology: a 2-year evaluation of 'hot clinics' in a tertiary neuroscience centre
}

\author{
Authors: Benjamin Ramsahoye, ${ }^{A}$ Sam Massias, ${ }^{A}$ Pablo Garcia Reitboeck, ${ }^{B}$ Kuven Moodley ${ }^{B}$ and Bhavini Patel ${ }^{B}$
}

\section{Introduction}

Patients with neurological symptoms account for $10-20 \%$ of acute medical admissions. '. Early neurologist contact for this cohort improves diagnostic outcomes, reduces length of stay and avoids diagnostic procedures. ${ }^{2}$ The rapid access neurology clinics ('hot' clinics) at St George's Hospital (SGH), London, are part of an innovative hyperacute neurology service that aims to reduce the burden of unnecessary admissions to the acute medical take. ${ }^{2}$ Future-proofing of such services require a proactive approach to how they are being utilised. We analysed this in greater detail.

We analysed referrals to the hot clinics from August 2016 to July 2018 , assessing the performance of the second year compared with the first. We then identified inappropriate referrals to the clinic to assess how resources might be optimised in the future. This will be of relevance to others who wish to replicate our model.

\section{Materials and methods}

Two years of hot clinic appointments between 1 August 2016 and 31 July 2018 were analysed. This included final diagnosis, appointment waiting times, time taken to reach a final diagnosis and the number of hospital admissions avoided.

\section{Results and discussion}

A total of 760 patients were reviewed over 2 years. In that period, general practitioner (GP) referrals increased by more than $200 \%$. The commonest diagnoses were migraine, functional neurological disorder (FND), neuro-inflammatory disorders (including multiple sclerosis), other primary headache disorders, and compressive radiculopathy (Fig 1).

Thirty-eight per cent of referrals were headache-related, of which $16.9 \%$ were migraine. Chronic migraine represented $20.1 \%$ of the migraine cohort (75\% from the emergency department (ED)). Of headache patients, $54.2 \%(n=155)$ had an MRI, of which only five were abnormal.

Compared with year 1 , there was an increase in hot clinic appointments from 232 to 528 in year 2. In year 2, 51.5\% of patients were referred from primary care compared with $30.6 \%$ in year 1 ( $p<0.001$, Fisher's exact test; Fig 2$)$.

Authors: A St George's, University of London, London, UK; ${ }^{\text {B St }}$ George's University Hospitals NHS Foundation Trust, London, UK

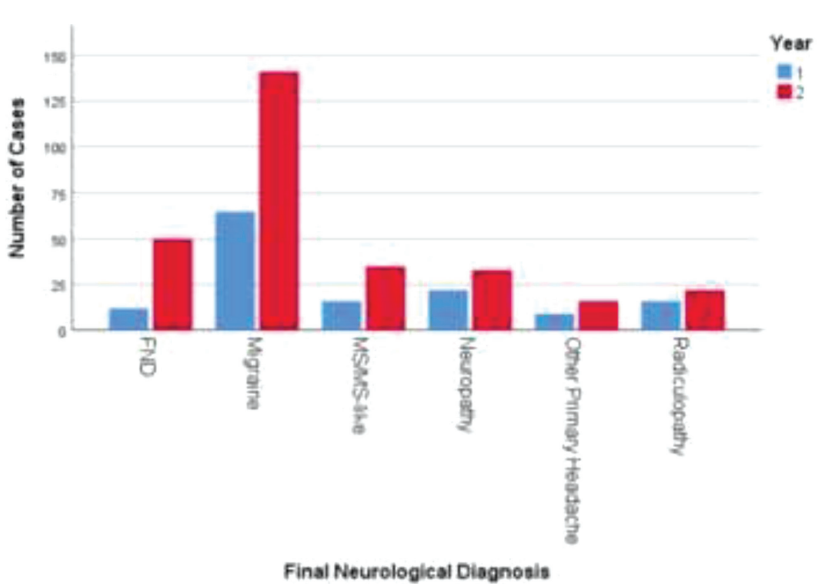

Fig 1. The most common neurological diagnoses made in hot clinics over the 2-year period.

The median (interquartile range) waiting time for year 2 increased compared with year 1 (6.0 (2.0-8.0) year 2; 4.0 (2.0-7.0) year $1 ; p<0.001$, median test). The proportion of admissions avoided in year 2 decreased compared with year $1(56.8 \%$ vs $67.2 \%$ respectively; $p=0.008$, Fisher's exact test).

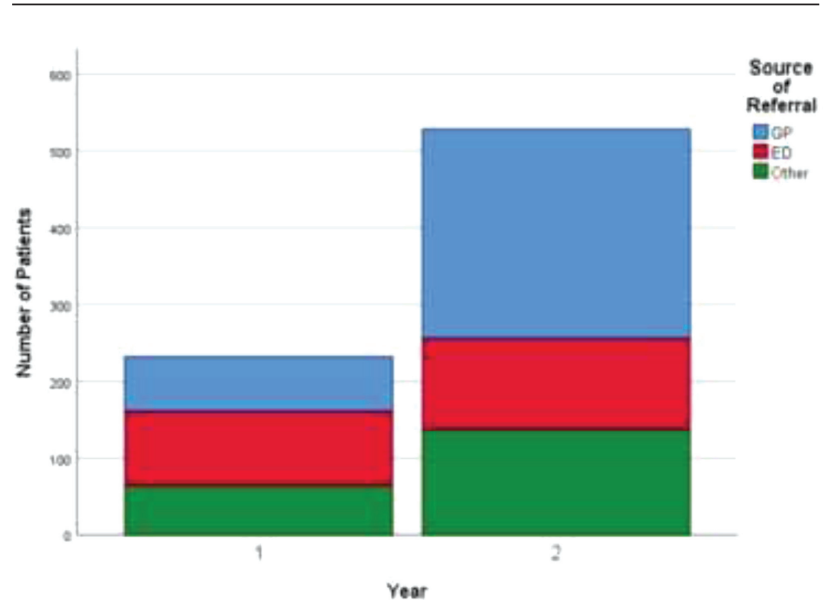

Fig 2. Sources of referral to hot clinics for patients in year 1 and year 2 . 


\section{Conclusions}

The use of this service has grown significantly over time. However, a significant proportion of this growth is represented by chronic migraine and compressive radiculopathy. Given the natural history of these conditions, neither are suitable for hot clinic referral. In particular, chronic migraine and other primary headaches are poorly recognised by referrers. Reducing inappropriate referrals of this nature is important to prevent decline in the quality of service (waiting times, admissions avoided). This is critically important should such clinics become the standard for acute ambulatory neurology care, in particular for patients discharged from ED/ acute medicine. One solution would be to encourage GPs to discuss prospective neurological admissions directly with a neurologist (rather than the medical registrar on call, as is standard). Another would be to encourage the ED to direct a larger proportion of their ambulatory neurological patients to hot clinics rather than acute medicine once suitable criteria are agreed, in turn supported by adequate education of non-neurologists regarding excluded conditions.

\section{Conflicts of interest}

None declared.

\section{References}

1 Gregory R, Nicholl D, Lawrence J. Association of British Neurologists: Acute neurology services survey 2017. ABN, 2017. www.theabn.org/ resource/collection/219B4A48-4D25-4726-97AA-0EB6090769BE/ ABN_2017_Acute_Neurology_Survey.pdf [Accessed 07 February 2020].

2 Moodley KK, Jones V, Yogarajah M et al. Hyperacute neurology at a regional neurosciences centre: a 1-year experience of an innovative service model. Clin Med 2019;19:119-26. 